\title{
Green's functions, Biot-Savart operators, and linking numbers on negatively curved symmetric spaces
}

Cite as: J. Math. Phys. 60, 111503 (2019); https://doi.org/10.1063/1.5109244

Submitted: 07 May 2019 • Accepted: 09 October 2019 • Published Online: 04 November 2019

Stefan Bechtluft-Sachs and Evangelia Samiou

\section{ARTICLES YOU MAY BE INTERESTED IN}

Orthogonal polynomials, asymptotics, and Heun equations

Journal of Mathematical Physics 60, 113501 (2019); https://doi.org/10.1063/1.5102102

Evolutionary equations and constraints: Maxwell equations

Journal of Mathematical Physics 60, 113503 (2019); https://doi.org/10.1063/1.5109087

On the mean field limit for Brownian particles with Coulomb interaction in 3D

Journal of Mathematical Physics 60, 111501 (2019); https://doi.org/10.1063/1.5114854

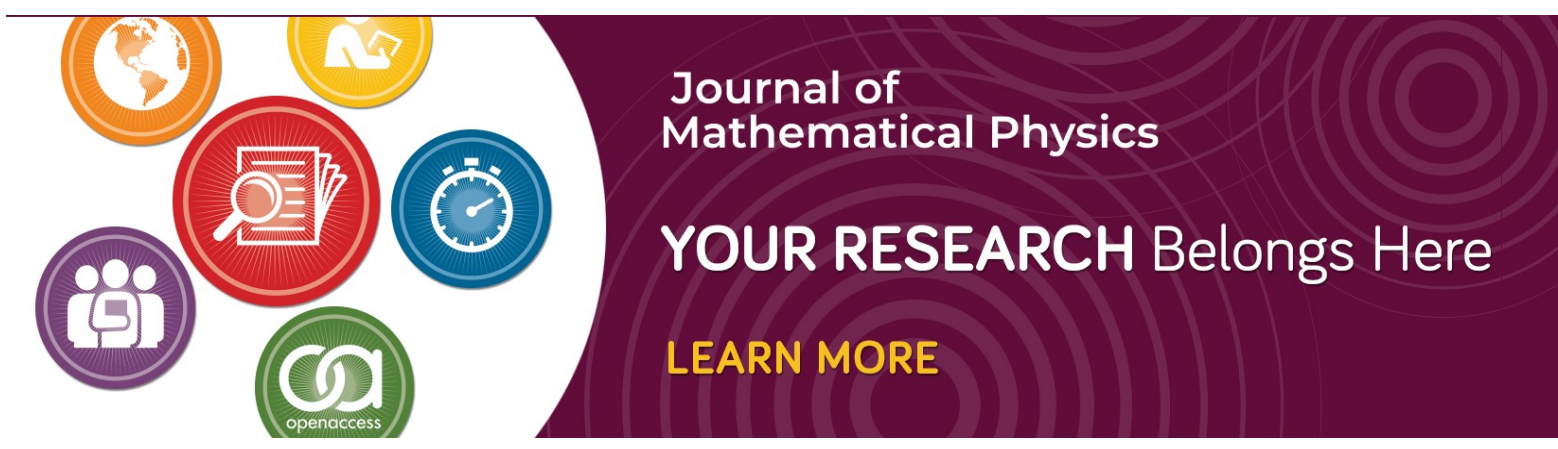




\title{
Green's functions, Biot-Savart operators, and linking numbers on negatively curved symmetric spaces
}

\author{
Cite as: J. Math. Phys. 60, 111503 (2019); doi: 10.1063/1.5109244 \\ Submitted: 7 May 2019 • Accepted: 9 October 2019 • \\ Published Online: 4 November 2019
}

\section{Stefan Bechtluft-Sachs ${ }^{1, a)}$ and Evangelia Samiou ${ }^{2, b)}$}

\section{AFFILIATIONS}

${ }^{1}$ Department of Mathematics and Statistics, Maynooth University, Maynooth, Ireland

${ }^{2}$ Department of Mathematics and Statistics, University of Cyprus, Nicosia, Cyprus

a) Electronic mail: stefan.bechtluft-sachs@mu.ie

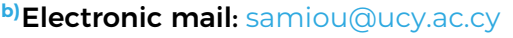

\section{ABSTRACT}

We construct radial fundamental solutions for the differential form Laplacian on negatively curved symmetric spaces. At least, one of these Green's functions also yields a Biot-Savart operator, i.e., a right inverse of the exterior differential on closed forms with image in the kernel of the codifferential. Any Biot-Savart operator gives rise to a Gauss linking integral.

Published under license by AIP Publishing. https://doi.org/10.1063/1.5109244

\section{INTRODUCTION}

The purpose of this paper is to extend the well-known radial analysis of the scalar Laplace operator on the Euclidean space $\mathbb{R}^{m}$ to the differential form Laplacian on negatively curved symmetric spaces. In more detail, in $\mathbb{R}^{m}$, by rotational symmetry, Poisson's equation reduces to an ordinary differential equation. For this reduction, the key notion is that of a radial kernel function. For example, the electrostatic potential $\phi$ caused by a charge distribution $\rho$ must satisfy the Poisson equation,

$$
\Delta \phi=\rho .
$$

If $\rho$ has compact support, then there is a radial solution,

$$
\phi(x)=[Q \rho](x)=\int_{\mathbb{R}^{3}} \frac{\rho(y)}{4 \pi\|x-y\|} d^{3} y .
$$

Thus, the scalar Laplacian $\Delta$ has a right inverse $Q$ with integral kernel $q(x, y)=A(d(x, y))$ depending on the distance only, and Poisson's equation becomes equivalent to the ordinary differential equation,

$$
A^{\prime \prime}(t)+\frac{2}{t} A^{\prime}(t)=0 \quad \text { with } \quad A(t) \sim \frac{-1}{t \mathbf{v o l} S^{2}} \text { as } t \rightarrow 0,
$$

which has the solution $A(t)=\frac{-1}{4 \pi t}$. This generalizes to harmonic spaces, i.e., Riemannian manifolds whose distance spheres have constant mean curvature. All negatively curved symmetric spaces are harmonic (see Sec. II C).

We will extend this construction of a fundamental solution to the differential form Laplace operator to negatively curved symmetric spaces $X$. For a given compactly supported $l$-form, $\omega \in \Omega_{c}^{l}(X)$, we look for a solution $\mu \in \Omega^{l}(X)$ of $\Delta \mu=\omega$ in the form

$$
\mu_{x}=[Q \omega]_{x}=\int_{X} q_{x, y} \omega_{y} d y .
$$


The difficulty here is that the kernel function $q$ of the integral operator $Q$ in (3) consists of maps $q_{x, y}: \Lambda^{l} T_{y}^{*} X \rightarrow \Lambda^{l} T_{x}^{*} X$ relating different fibers of the bundle $\Lambda^{l} T^{*} X$ of alternating forms, i.e., in general different vector spaces. To reduce the partial differential equation for the integral kernel $q$ in (3) to an ordinary differential equation and fully exploit the two point symmetry of $X$, we need a reference map. In this paper, we will use the parallel transport, i.e., we will compare $q_{x, y}$ with the parallel transport along the unique geodesic from $y$ to $x$. Our main Theorem 1 contains the radial equation (22), in "parallel transport form," in the terminology of Refs. 5 and 6, for the differential form Laplacian. Radial Green's functions for the Laplacian on differential forms exist and are determined by solutions of a matrix valued ordinary differential equation resembling (2) with the factor $\frac{1}{t}$ replaced by the mean curvature of the distance spheres and a more involved right hand side reflecting the more complicated curvature of $X$.

Hodge theory provides a close link between Green's functions for the differential form Laplacian, Biot-Savart operators, and Gauss linking integrals on compact Riemannian manifolds. In the noncompact case, this no longer holds. Nonetheless, in Theorem 3, we show that there is choice of a solution $A$ of the radial equation (22) so that the right inverse $Q^{A}$ of $\Delta$ corresponding to $A$ gives a Biot-Savart operator with radial kernel. In Theorem 4, this leads to a Gauss integral formula for the linking number in negatively curved symmetric spaces.

For 3-dimensional space forms, Refs. 3, 5, and 6 obtained explicit formulas for the differential form Green's function, the Biot-Savart operator, and Gauss linking integrals by more direct means. The expressions of $q$ in terms of elementary functions are quite intricate, already in this case. In Sec. III B, we derive the explicit radial equation for the Green's function in hyperbolic space and compare it with the result of Ref. 5.

While the the Biot-Savart operator solves the Cartan differential and the codifferential simultaneously, a Gauss intergral for the linking number can be obtained by solving the differential only. In space forms, the linking number can also be expressed as a mapping degree. The resulting explicit formulas are given in Ref. 7 and also in Ref. 11.

\section{PRELIMINARIES}

We recall some fundamental facts for the Laplace operator on differential forms and its inversion in $\mathbb{R}^{m}$ by means of the Newton potentials. Then, we discuss the consequences of fixing an axis in a rank one symmetric space, in particular the reduction of equivariant kernel functions to functions on $\mathbb{R}^{+}$.

For the Laplace operator on forms, we refer to Refs. 1 and 12. For symmetric spaces, see Refs. 9, 10, and 14.

\section{A. The Weitzenböck formula}

The Laplace operator on differential forms on a Riemannian manifold $X^{m}$ is defined by

$$
\Delta \omega=\left(d d^{*}+d^{*} d\right) \omega
$$

where $d: \Omega^{k}(X) \rightarrow \Omega^{k+1}(X)$ denotes the exterior differential and $d^{*}$ its adjoint. In terms of an orthonormal basis $e_{1}, \ldots, e_{m}$ of $T_{p} X$, these are given by

$$
d \omega=e_{\mu} \wedge \nabla e_{\mu} \omega, \quad d^{*} \omega=-e_{\mu} \neg \nabla e_{\mu} \omega,
$$

where $\nabla$ is the Levi-Civita connection and for $\alpha \in \Omega^{l}(X)$,

$$
\begin{gathered}
e_{\mu} \wedge \alpha\left(x_{0}, x_{1}, \ldots, x_{l}\right)=\sum_{j=0}^{l}(-1)^{j}\left\langle e_{\mu} \mid x_{j}\right\rangle \alpha\left(x_{0}, x_{1}, \ldots, \hat{x}_{j}, \ldots, x_{l}\right), \\
e_{\mu} \neg \alpha\left(x_{1}, \ldots, x_{l-1}\right)=\alpha\left(e_{\mu}, x_{1}, \ldots, x_{l-1}\right) .
\end{gathered}
$$

By the Weitzenböck formula,

$$
\Delta=\nabla^{*} \nabla+\mathscr{R}^{*}
$$

where

$$
\nabla^{*} \nabla \omega=-\operatorname{trace}\left(\nabla^{2} \omega\right)
$$

and

$$
\mathscr{R}^{*}=-\sum_{\mu, v=1}^{m} e_{\mu} \wedge e_{v} \neg R_{e_{\mu}, e_{v}} .
$$


Thus, if $\omega \in \Omega^{l+1}(X)$, then $\mathscr{R}^{*} \omega=\omega \circ \mathscr{R}$ with

$$
\mathscr{R}\left(v_{0} \wedge \ldots \wedge v_{l}\right)=\sum_{i=0}^{l} v_{0} \wedge \ldots \wedge \operatorname{Ric}_{i} v_{i} \wedge \ldots \wedge v_{l}+\sum_{0 \leq j<i \leq l} \sum_{\substack{v=1 \\ m}}^{m} v_{0} \wedge \ldots \wedge R_{v_{i}, v_{j}} e_{v} \wedge \ldots \wedge e_{\nu} \ldots \wedge v_{l} .
$$

In low degrees 0,1 , and 2 , this becomes

$$
\begin{aligned}
& l+1=0: \\
& l+1=1: \\
& l+1=2:
\end{aligned}
$$$$
\mathscr{R}=0,
$$$$
\mathscr{R}(v)=\sum_{v} R_{v, e_{v}} e_{v}=\operatorname{Ric}(v),
$$

$$
\mathscr{R}(v \wedge w)=\operatorname{Ric}(v) \wedge w+v \wedge \operatorname{Ric}(w)+\sum_{v} R_{w, v} e_{v} \wedge e_{v} .
$$

\section{B. The Newton potential for differential forms on $\mathbb{R}^{m}$}

On $\mathbb{R}^{m}$, the Laplace operator on forms takes a simple form. For $0 \leq l \leq m$, a $l$-form can be written as

$$
\alpha=\sum_{1 \leq i_{1} \leq \cdots \leq i_{l} \leq m} a_{i_{1}, \cdots i_{l}} d x_{i_{1}} \wedge d x_{i_{2}} \wedge \ldots \wedge d x_{i_{l}}
$$

with $a_{i_{1}, \cdots i_{l}} \in C^{\infty}\left(\mathbb{R}^{m}\right)$, where the $x_{i}$ denote the standard coordinates. A straightforward calculation shows that the Laplace operator acts componentwise,

$$
\Delta \alpha=\sum_{1 \leq i_{1} \leq \cdots \leq i_{l} \leq m}\left(\Delta a_{i_{1}, \cdots i_{l}}\right) d x_{i_{1}} \wedge d x_{i_{2}} \wedge \ldots \wedge d x_{i_{l}} .
$$

The Newton potentials are a Green's function for the Laplacian on functions. They are defined by

$$
N(x, y):=\left\{\begin{array}{cc}
\frac{1}{(m-2)\|x-y\|^{m-2} \operatorname{vol} S^{m-1}} & \text { if } m \neq 2 \\
\frac{-\log (\|x-y\|)}{2 \pi} & \text { if } m=2 .
\end{array}\right.
$$

If $\omega \in \Omega^{l}\left(\mathbb{R}^{m}\right)$ is a differential $l$-form on $\mathbb{R}^{m}$ of compact support, then

$$
\Delta_{x} \int_{\mathbb{R}^{m}} N(x, y) \omega_{y} d^{m} y=\omega
$$

If $\omega$ is closed then a solution $\alpha$ to the Cartan equation $d \alpha=\omega$ on $\mathbb{R}^{m}$ is given by

$$
\alpha_{x}=d_{x}^{*} \int_{\mathbb{R}^{m}} N(x, y) \omega_{y} d^{m} y=\int_{\mathbb{R}^{m}} \frac{(x-y) \neg \omega_{y}}{\mathbf{v o l} S^{m-1}\|x-y\|^{m}} d^{m} y .
$$

In the case $m=3$, this is the Biot-Savart formula for the magnetic field $\alpha$ caused by a stationary current $\omega$.

\section{Harmonic spaces}

Harmonic spaces are Riemannian manifolds whose volume form in normal coordinates is a function of the distance from the origin. This is equivalent to all distance spheres of radius $t$ having constant mean curvature $h(t)$, a function of the radius only. A consequence is that the scalar Laplace operator has a Green's function $q(x, y)$, which is a radial kernel function in the sense of Ref. 15, i.e., there is a function $A$ so that $q(x, y)=A\left(d(x, y)\right.$. Rank one symmetric spaces are harmonic. The converse, the famous Lichnerowicz conjecture, ${ }^{13}$ was proved by Szabo ${ }^{15}$ for compact spaces. There are, however, noncompact, nonsymmetric harmonic Riemannian manifolds (Damek and Ricci spaces $\left.^{4}\right)$.

Let $h(t)$ be the mean curvature of the spheres of radius $t$ in a $m$-dimensional harmonic space $X, m>2$, and let $A: \mathbb{R}^{+} \rightarrow \mathbb{R}$ solve the ordinary differential equation,

$$
A^{\prime \prime}(t)+h(t) A^{\prime}(t)=0 \quad \text { with } \quad A(t) \sim \frac{1}{(m-2) t^{m-2} \mathbf{v o l} S^{m-1}} \text { as } t \rightarrow 0 .
$$


Then, the integral operator $Q$ defined by

$$
[Q \rho](x)=\int_{X} A(d(x, y)) \rho(y) d y
$$

for compactly supported functions $\rho$ on $X$ is a right inverse of the scalar Laplacian, i.e., $\Delta Q \rho=\rho$. In (9) and in the sequel, integration over $X$ is always with respect to the Riemannian volume.

\section{Negatively curved symmetric spaces}

Negatively curved symmetric spaces are exactly the noncompact rank one symmetric spaces. These are two point homogeneous, i.e., the isometry group acts transitively on equidistant pairs. In particular, these spaces are harmonic.

Let $X$ be a rank one symmetric space of dimension $m=n+1$. We fix a point $p \in X$ and a unit tangent vector $T(p) \in S T_{p} X$. Let $G$ be the group of isometries of $X$, and let $K=G_{p}$ be the isotropy group at $p$. Let $H=G_{p, T} \subset K$ be the isotropy group of the vector $T$,

$$
H=\{g \in G \mid g p=p \text { and } d g T=T\} .
$$

We will identify the Lie algebra $\mathfrak{g}$ of $G$ with that of the Killing fields of $X$. The Lie algebras of $K$ and $H$ correspond to spaces of Killing fields,

$$
\begin{aligned}
\mathfrak{k} & =\{k \in \mathfrak{g} \mid k(p)=0\}, \\
\mathfrak{h} & =\left\{h \in \mathfrak{k} \mid \nabla_{T} h(p)=0\right\}=\{h \in \mathfrak{k} \mid[T, h]=0\},
\end{aligned}
$$

where we have identified $T$ with its extension to a Killing field in

$$
\mathfrak{m}=\{m \in \mathfrak{g} \mid \nabla m(p)=0\} .
$$

The scalar product on $\mathfrak{m}$ will be so that the isomorphism

$$
\mathfrak{m} \rightarrow T_{p} X, \quad m \mapsto m(p)
$$

is isometric. Let $\mathfrak{m}_{0}$ be the orthogonal complement of $T$ in $\mathfrak{m}$. Since $X$ is symmetric of rank one, the map

$$
\begin{aligned}
& \mathfrak{k} \rightarrow \mathfrak{s o}(\mathfrak{m}) \rightarrow \mathfrak{m}_{0}, \\
& k \mapsto \operatorname{ad}_{k} \mapsto[T, k]
\end{aligned}
$$

is surjective and has kernel $\mathfrak{h}$. If $X$ is not flat, i.e., not a Euclidean space, then

$$
\mathfrak{k}_{0}:=\left[T, \mathfrak{m}_{0}\right]
$$

is a complement to $\mathfrak{h}$ in $k$. In case $X=\mathbb{R}^{n}$, we let $\mathfrak{k}_{0}$ be the set of infinitesimal generators of rotations in planes containing $T$. In any case, we obtain splittings of $\mathfrak{g}$, invariant under $A d_{K}$, respectively, $A d_{H}$,

$$
\mathfrak{g}=\mathfrak{k} \oplus \mathfrak{m}=\underbrace{\mathfrak{h} \oplus \mathfrak{k}_{0}}_{=\mathfrak{k}} \oplus \underbrace{\langle T\rangle \oplus \mathfrak{m}_{0}}_{=\mathfrak{m} \cong T_{p} X},
$$

and an isomorphism

$$
\mathfrak{k}_{0} \rightarrow \mathfrak{m}_{0}, \quad k \mapsto[T, k]
$$

Below, we list $\mathfrak{k}, \mathfrak{h}$ and the decomposition of $\mathfrak{m}$ into irreducible $H$-modules for the rank one symmetric spaces.

\begin{tabular}{lccc}
\hline \hline$X$ & $\mathfrak{k}$ & $\mathfrak{h}$ & $\mathfrak{m}=\langle T\rangle \oplus \mathfrak{m}_{0}$ \\
\hline $\mathbb{R}^{n}, S^{n}, H^{n}$ & $\mathfrak{s o}(n)$ & $\mathfrak{s o}(n-1)$ & $\langle T\rangle \oplus \mathbb{R}^{n-1}$ \\
$\mathbb{C} \mathrm{P}^{n}, \mathbb{C} \mathrm{H}^{n}$ & $\mathfrak{u}(n)$ & $\mathfrak{u}(n-1)$ & $\langle T\rangle \oplus\langle i T\rangle \oplus \mathbb{C}^{n-1}$ \\
$\mathbb{H} \mathrm{P}^{n}, \mathbb{H} \mathrm{H}^{n}$ & $\mathfrak{s p}(1) \oplus \mathfrak{s p}(n)$ & $\mathfrak{s p}(1) \oplus \mathfrak{s p}(n-1)$ & $\langle T\rangle \oplus\langle i T, j T, k T\rangle \oplus \mathbb{H}^{n-1}$ \\
$\mathbb{O} \mathrm{P}^{2}, \mathbb{O} \mathrm{H}^{2}$ & $\mathfrak{s o}(9)=\mathfrak{s p i n}(9)$ & $\mathfrak{s o}(7)=\mathfrak{s p i n}(7)$ & $\langle T\rangle \oplus \mathbb{R}^{7} \oplus \mathbb{O}$ \\
\hline \hline
\end{tabular}


Because of the Jacobi identity, the operator $J: \mathfrak{m} \rightarrow \mathfrak{m}, J(m)=[T,[T, m]]$ is symmetric. In fact, for $n, m \in \mathfrak{m}$, we have

$$
\begin{aligned}
\langle[T,[T, m]] \mid n\rangle & =-\langle T \mid[n,[T, m]]\rangle \\
& =-\langle T \mid[[n, T], m]\rangle-\underbrace{\langle T \mid[T,[n, m]]\rangle}_{=0} \\
& =-\langle T \mid[m,[T, n]]\rangle,
\end{aligned}
$$

because $[\mathfrak{m}, \mathfrak{m}] \subset \mathfrak{k}$ and $\operatorname{ad}_{k} \in \mathfrak{s o}(\mathfrak{m})$ for all $k \in \mathfrak{k}$.

The geodesic $\gamma$ with $\gamma(0)=p$ and $\gamma^{\prime}(0)=T$ coincides with the one parameter orbit generated by $T$, i.e., $\gamma(t)=e^{t T} p$ and $T$ is the generator of the one parameter group of translations along $\gamma$. At the point $p$, the operator $J$ coincides with the Jacobi operator along the geodesic, i.e., $J m(p)=R_{T, m} T(p)$. In the usual normalization of the metric on $X$, it has eigenvalues 0 if $X=\mathbb{R}^{m},-1,-4$ if $X$ is of compact type, and 1,4 if $X$ is of noncompact type.

Lemma 1. Let $m \in \mathfrak{m}$ be an eigenvector of $J, J m=[T,[T, m]]=\lambda^{2} m$ with $\lambda \neq 0$. Let $k=\frac{1}{\lambda^{2}}[T, m] \in \mathfrak{k}$. Then,

$$
\begin{gathered}
\nabla_{T} k(p)=m(p), \\
{[T, k]=m \text { and }[m, k]=-T .}
\end{gathered}
$$

In particular, $a d_{k}: x \mapsto[x, k]$ preserves the plane spanned by $T$ and $m$.

Proof. The first equation in (13) holds by assumption, and since $[T, k]=\nabla_{T} k-\nabla_{k} T$ and $k(p)=0$, this implies (12).

It suffices to show the second equation at the point $p$ since both sides are Killing fields in $\mathfrak{m}$. On the unit sphere $S \mathfrak{m} \subset \mathfrak{m}$, consider the function $S \mathfrak{m} \rightarrow \mathbb{R}, x \mapsto\langle[m,[x, m]] \mid x\rangle=\left\langle R_{x, m} m \mid x\right\rangle$. At $x=T$, this takes an extreme value; hence, $T$ is critical. It follows that $\langle[m,[T, m]] \mid x\rangle=0$, whenever $x \perp T$; hence, $[m,[T, m]]=\lambda^{2} T$.

Notation 2. We fix an orthonormal basis $\left\{m_{i} \mid i=1 \ldots n\right\}$ of $\mathfrak{m}_{0}$ consisting of eigenvectors of $J$, i.e., so that $J m_{i}=\lambda_{i}^{2} m_{i}\left(i . e ., R_{T, m_{i}} T=\lambda_{i}^{2} m_{i}\right)$. If $X$ is not flat, we let $k_{i}=\frac{1}{\lambda_{i}^{2}}\left[T, m_{i}\right] \in \mathfrak{k}_{0}$. If $X=\mathbb{R}^{m}$, we let $k_{i}$ be the generator of the rotation in the plane spanned by $T$ and $m_{i}$. In all cases, we have a basis $\left\{k_{i} \mid i=1 \ldots n\right\}$ of $\mathfrak{k}_{0}$ so that (12) and (13) hold.

For $x, y \in X$, we denote by $P_{x, y}: T_{y} X \rightarrow T_{x} X$ the parallel transport from $y$ to $x$ along the minimizing geodesic. Thus, $P_{p, \gamma(t)}$ denotes the parallel transport along $\gamma$ from $\gamma(t)$ to $p=\gamma(0)$. Since $X$ is symmetric, the Jacobi operator $V \mapsto R_{T V} T$ along $\gamma$ commutes with the parallel transport along $\gamma$, i.e.,

$$
R_{T, P_{\gamma(t), p}} T=P_{\gamma(t), p} R_{T V} T=P_{\gamma(t), p} J V
$$

for $V \in T_{p} X$. The Killing fields $k \in \mathfrak{k}$ are Jacobi fields along the geodesic $\gamma$ satisfying the second order differential equation

$$
\nabla_{T} \nabla_{T} k(\gamma(t))=R_{T, k(\gamma(t))} T=P_{\gamma(t), p} J P_{p, \gamma(t)} k
$$

If $m(p)=\nabla_{T} k(p)$ is an eigenvector of $J$ corresponding to the eigenvalue $\lambda^{2}, \lambda \in i \mathbb{R}$ or $\lambda \in \mathbb{R}$, then

$$
k(\gamma(t))=P_{\gamma(t), p} \frac{\sinh (\lambda t)}{\lambda} m(p) \quad \text { and } \quad \nabla_{T} k(\gamma(t))=m(\gamma(t))=P_{\gamma(t), p} \cosh (\lambda t) m(p) .
$$

From these formulas, we immediately compute the volume $\sigma(t)$ of the geodesic sphere of radius $t$ and its mean curvature $h(t)$,

$$
\begin{aligned}
& \sigma(t)=\operatorname{vol}\left(S^{n}\right) \prod_{i=1}^{n} \frac{\sinh \left(\lambda_{i} t\right)}{\lambda_{i}}=t^{n}\left(\operatorname{vol}\left(S^{n}\right)+\frac{t^{2}}{6} \sum_{i=1}^{n} \lambda_{i}^{2}+\cdots\right), \\
& h(t)=\frac{\sigma^{\prime}(t)}{\sigma(t)}=\sum_{i=1}^{n} \frac{\lambda_{i} \cosh \left(\lambda_{i} t\right)}{\sinh \left(\lambda_{i} t\right)}=\frac{n}{t}+\frac{t}{3} \sum_{i=1}^{n} \lambda_{i}^{2}+\cdots .
\end{aligned}
$$

We will need to compute $\nabla_{k} k^{\prime}$ for basis vectors $k, k^{\prime} \in \mathfrak{k}_{0}$ along $\gamma$. 
Lemma 3. Let $m, m^{\prime} \in \mathfrak{m}_{0}$ be elements of the basis chosen in 2, with corresponding $k, k^{\prime} \in \mathfrak{k}_{0}$ and eigenvalues $\lambda, \lambda^{\prime}$. Then,

$$
\nabla_{m} k^{\prime}(\gamma(t))=\cosh (\lambda t) \cosh \left(\lambda^{\prime} t\right) P_{\gamma(t), p} \operatorname{ad}_{k^{\prime}}(m)(p) .
$$

In particular,

$$
\nabla_{u} k(\gamma(t))=\cosh (\lambda t) P_{\gamma(t), p} \mathrm{ad}_{k} P_{p, \gamma(t)} u
$$

for every $u \in T_{\gamma(t)} X$ and

$$
\nabla_{k} k=-\frac{1}{\lambda} \sinh (\lambda t) \cosh (\lambda t) T .
$$

Proof. Killing fields $X$ in a Riemannian manifold satisfy the differential equation

$$
R_{A, X} B=\nabla_{A, B}^{2} X
$$

for any vector fields $A, B$. For $u, v, w \in \mathfrak{m}$, we also have

$$
R_{v, w} u(p)=[u,[v, w]](p)
$$

(see Ref. 9, for example). By (14) and since $R$ is parallel,

$$
\begin{aligned}
R_{T, k^{\prime}} m(\gamma(t)) & =R_{T, P_{\gamma(t), p} m^{\prime}(p) \frac{\sinh \left(\lambda^{\prime} t\right)}{\lambda^{\prime}}} P_{\gamma(t), p} m(p) \cosh (\lambda t) \\
& =\frac{\sinh \left(\lambda^{\prime} t\right) \cosh (\lambda t)}{\lambda^{\prime}} P_{\gamma(t), p} R_{T, m^{\prime}} m(p) \\
& =\frac{\sinh \left(\lambda^{\prime} t\right) \cosh (\lambda t)}{\lambda^{\prime}} P_{\gamma(t), p}\left[m,\left[T, m^{\prime}\right]\right](p) \\
& =\lambda^{\prime} \sinh \left(\lambda^{\prime} t\right) \cosh (\lambda t) P_{\gamma(t), p}\left[m, k^{\prime}\right](p) \\
& =\lambda^{\prime} \sinh \left(\lambda^{\prime} t\right) \cosh (\lambda t) P_{\gamma(t), p} \nabla_{m} k^{\prime}(p) .
\end{aligned}
$$

On the other hand, by the differential equation for Killing fields, we can compute the same as

$$
\begin{aligned}
R_{T, k^{\prime}} m(\gamma(t)) & =\nabla_{T, m}^{2} k^{\prime}(\gamma(t)) \\
& =\nabla_{T} \nabla_{m} k^{\prime}(\gamma(t))-\nabla_{\nabla_{T} m} k^{\prime}(\gamma(t)) \\
& =\nabla_{T} \nabla_{m} k^{\prime}(\gamma(t))-\frac{\lambda \sinh (\lambda t)}{\cosh (\lambda t)} \nabla_{m} k^{\prime}(\gamma(t)) .
\end{aligned}
$$

Thus, $\nabla_{m} k^{\prime}(\gamma(t))$ satisfies the differential equation

$$
\lambda^{\prime} \sinh \left(\lambda^{\prime} t\right) \cosh (\lambda t) P_{\gamma(t), p} \nabla_{m} k^{\prime}(p)=\nabla_{T} \nabla_{m} k^{\prime}(\gamma(t))-\frac{\lambda \sinh (\lambda t)}{\cosh (\lambda t)} \nabla_{m} k^{\prime}(\gamma(t)),
$$

which has the unique solution

$$
\nabla_{m} k^{\prime}(\gamma(t))=\cosh (\lambda t) \cosh \left(\lambda^{\prime} t\right) P_{\gamma(t), p} \nabla_{m} k^{\prime}(p)
$$

\section{E. Equivariant Kernel functions}

Let

$$
Q: \Omega^{l}(X) \rightarrow \Omega^{k}(X)
$$

be an integral operator, i.e.,

$$
(Q \omega)_{x}=\int_{X} \hat{q}_{x, y} \omega_{y} d y
$$


where $\hat{q}$ is a section in the exterior homomorphism bundle,

$$
\operatorname{Hom}\left(\Lambda^{l} T X^{*}, \Lambda^{k} T X^{*}\right) \rightarrow X \times X
$$

i.e., $\hat{q}_{x, y} \in \operatorname{Hom}\left(\Lambda^{l} T_{x} X^{*}, \Lambda^{k} T_{y} X^{*}\right) \cong \operatorname{Hom}\left(\Lambda^{k} T_{y} X, \Lambda_{x}^{l} T X\right)$. We can rewrite $\hat{q}$ in the form $\hat{q} \omega=\omega \circ q$ with some section,

$$
q \in \Gamma \operatorname{Hom}\left(\Lambda^{k} T X, \Lambda^{l} T X\right)
$$

hence,

$$
(Q \omega)_{x}=\int_{X} \omega_{y} \circ q_{y, x} d y
$$

The operator $Q$ is $G$-equivariant if and only if

$$
q_{y, x}=g^{-1} q_{g y, g x} g
$$

for all $x, y \in X, g \in G$. Rank one symmetric spaces are two point homogeneous. We can, therefore, map any pair $x, y \in X$ to the geodesic $\gamma$ by an isometry $g$ of $X$ so that

$$
g y=p=\gamma(0), \quad g x=\gamma(t),
$$

where $t=d(x, y) \in \mathbb{R}_{0}^{+}$is the distance of the two points. The parallel transport satisfies the same equivariance (18) as $q$. We can, therefore, rewrite $q=q^{A}$ in the form

$$
q_{y, x}^{A}=g^{-1} q_{\gamma(0), \gamma(t)}^{A} g=g^{-1} A(t) P_{\gamma(0), \gamma(t)} g
$$

with a function

$$
A: \mathbb{R}^{+} \rightarrow \operatorname{Hom}_{H}\left(\Lambda^{k} T_{\gamma(0)} X, \Lambda^{l} T_{\gamma(0)} X\right)=\operatorname{Hom}_{H}\left(\Lambda^{k} \mathfrak{m}, \Lambda^{l} \mathfrak{m}\right) .
$$

This establishes a one to one correspondence between equivariant kernel functions, respectively, equivariant integral operators on one side and functions on $\mathbb{R}^{+}$taking values in $\operatorname{Hom}_{H}\left(\Lambda^{k} \mathfrak{m}, \Lambda^{l} \mathfrak{m}\right)$ on the other,

$$
\begin{aligned}
\left(\left.\Gamma \operatorname{Hom}\left(\Lambda^{k} T X, \Lambda^{l} T X\right)\right|_{X \times X \backslash \Delta X}\right)^{G} & \leftrightarrow C^{\infty}\left(\mathbb{R}^{+}, \operatorname{Hom}_{H}\left(\Lambda^{k} \mathfrak{m}, \Lambda^{l} \mathfrak{m}\right)\right), \\
q^{A} & \leftrightarrow A .
\end{aligned}
$$

In the case $l=k=0$, this is the bijection between radial kernel functions (see Ref. 15) and functions on the positive real line.

\section{A RIGHT INVERSE FOR THE LAPLACE OPERATOR ON FORMS}

In this section, we will construct an equivariant integral operator inverting the Laplacian. Thus, for $\omega \in \Omega^{l}(X)$ of compact support, we look for a solution $\mu \in \Omega^{l}(X)$ of

$$
\omega=\Delta \mu
$$

in the form

$$
\mu_{x}=\int_{X} \omega_{y} \circ q_{y, x}^{A} d y
$$

with an integral kernel determined by a function $A \in C^{\infty}\left(\mathbb{R}^{+}, \operatorname{Hom}_{H}\left(\Lambda^{l} \mathfrak{m}, \Lambda^{l} \mathfrak{m}\right)\right)$ as in (19).

Theorem 1. Let $\left\{k_{1}, \ldots, k_{n}\right\} \subset \mathfrak{k}_{0}$ be the basis of 2. Let $h(t)$ be the mean curvature of the geodesic sphere in $X$ of radius $t$. If $A \in$ $C^{\infty}\left(\mathbb{R}^{+}, \operatorname{Hom}_{H}\left(\Lambda^{l} \mathfrak{m}, \Lambda^{l} \mathfrak{m}\right)\right)$ is a solution of the ordinary second order differential equation,

$$
A^{\prime \prime}(t)+h(t) A^{\prime}(t)=A(t) \mathscr{R}-\sum_{i=1}^{n} \frac{\operatorname{ad}_{k_{i}}^{2} A(t)-2 \cosh \left(\lambda_{i} t\right) \operatorname{ad}_{k_{i}} A(t) \operatorname{ad}_{k_{i}}+\cosh \left(\lambda_{i} t\right)^{2} A(t) \operatorname{ad}_{k_{i}}^{2}}{\sinh \left(\lambda_{i} t\right)^{2} / \lambda_{i}^{2}}
$$

with initial condition

$$
A(t)=\frac{t^{1-n}}{(n-1) \mathbf{v o l} S^{n}}(1+o(1)) \quad \text { as } \quad t \rightarrow 0
$$


then $q^{A}$ as given in (19) is a Green's function for the Laplacian. Thus, for any $\omega \in \Omega^{l}(X)$ with compact support, we have

$$
\Delta_{x} \int_{X} \omega_{y} \circ q_{y, x}^{A} d y=\omega_{x}
$$

Note that the initial condition (23) on $A$ is so that $\omega_{p} \circ q_{p, x}^{A}$ is asymptotic to the Newton potential (6) in normal coordinates around $p$.

Proof. We will show that if $A$ satisfies (22) and (23), then $\Delta_{x}\left(\omega_{y} \circ q_{y, x}^{A}\right)=\delta_{y} \omega_{y}$ for all $y$ and $\omega_{y} \in \Lambda^{l} T_{y} X^{*}$, i.e.,

$$
\int_{X}\left\langle\Delta_{x} \alpha_{x} \omega_{y} \circ q_{y, x}^{A}\right\rangle d x=\left\langle\alpha_{y} \omega_{y}\right\rangle
$$

for all $\alpha \in \Omega^{l}(X)$ with compact support. By homogeneity, it suffices to do this for $y=p$ and $x=\gamma(t)$ for some $t \in \mathbb{R}_{0}^{+}$. To this end, let $\omega_{p} \in$ $\Lambda^{l} \mathfrak{m}^{*}=\Lambda^{l} T_{p} X^{*}$ and consider $\mu \in \Omega^{l}(X)$ given by

$$
\mu_{e^{s k} \gamma(t)}\left(v_{1} \wedge \ldots \wedge v_{l}\right)=\omega_{p}\left(e^{s k} A(t) P_{p, \gamma(t)} e^{-s k}\left(v_{1} \wedge \ldots \wedge v_{l}\right)\right)
$$

for $k \in \mathfrak{k}, s \in \mathbb{R}, t \in \mathbb{R}_{0}^{+}$, and $v_{i} \in T_{e^{s k} \gamma(t)} X$. Thus, $\mu$ is the composition

$$
\begin{aligned}
\mu_{e^{s k} \gamma(t)} & =\omega_{p} \circ q_{p, e^{s k} \gamma(t)}^{A}=\omega_{p} \circ e^{s k} \circ q_{p, \gamma(t)}^{A} e^{-s k} \\
& =\omega_{p} \circ e^{s k} A(t) P_{p, \gamma(t)} e^{-s k} .
\end{aligned}
$$

We now compute $\Delta \mu$ at $\gamma(t)$. From the Weitzenböck formula (5),

$$
\begin{aligned}
\Delta \mu(\gamma(t)) & =\left(-\nabla_{T, T}^{2} \mu-\sum_{i=1}^{n} \frac{1}{\left\|k_{i}\right\|^{2}} \nabla_{k_{i}, k_{i}}^{2} \mu+\mathscr{R}^{*} \mu\right)(\gamma(t)) \\
& =-\omega_{p} \circ A^{\prime \prime}(t) P_{p, \gamma(t)}+\mu \circ \mathscr{R}(\gamma(t))-\sum_{i=1}^{n} \frac{1}{\left\|k_{i}\right\|^{2}} \nabla_{k_{i}} \nabla_{k_{i}} \mu(\gamma(t))+\sum_{i=1}^{n} \frac{1}{\left\|k_{i}\right\|^{2}} \nabla_{\nabla_{k_{i}} k_{i}} \mu(\gamma(t)) .
\end{aligned}
$$

By (17),

$$
\sum_{i=1}^{n} \frac{1}{\left\|k_{i}\right\|^{2}} \nabla_{k_{i}} k_{i}(\gamma(t))=-h(t) T(\gamma(t))
$$

hence,

$$
\Delta \mu(\gamma(t))=-\omega_{p} \circ\left(A^{\prime \prime}(t)+h(t) A^{\prime}(t)\right) \circ P_{p, \gamma(t)}+\left(-\sum_{i=1}^{n} \frac{1}{\left\|k_{i}\right\|^{2}} \nabla_{k_{i}} \nabla_{k_{i}} \mu+\mu \circ \mathscr{R}\right)(\gamma(t)) .
$$

In order to compute $\nabla_{k} \nabla_{k} \mu$ for $k=k_{i} \in \mathfrak{k}_{0}$, we differentiate (25) for $s$,

$$
\left.\frac{D}{d s}\right|_{s=0}\left(e^{s k} A(t)\right)=\left.\frac{d}{d s}\right|_{s=0}\left(e^{s k} A(t)\right)=\operatorname{ad}_{k} A .
$$

For the calculation of $\left.\frac{D}{d s}\right|_{s=0} P_{p, \gamma(t)} e^{-s k}$, let $u=\left.\frac{d}{d r}\right|_{r=0} u(r) \in T_{\gamma(t)} X$. Then,

$$
\begin{aligned}
\left(\left.\frac{D}{d s}\right|_{s=0} P_{p, \gamma(t)} e^{-s k}\right) u & =\left.\left.\frac{D}{d s}\right|_{s=0} \frac{d}{d r}\right|_{r=0} P_{p, \gamma(t)} e^{-s k} u(r) \\
& =\left.\left.\frac{D}{d r}\right|_{r=0} \frac{d}{d s}\right|_{s=0} P_{p, \gamma(t)} e^{-s k} u(r) \\
& =-P_{p, \gamma(t)} \nabla_{u} k \\
& =-\cosh (\lambda t) \operatorname{ad}_{k} P_{p, \gamma(t)} u
\end{aligned}
$$


by (16). Hence,

$$
\nabla_{k} \mu(\gamma(t))=\omega_{p} \circ\left(\operatorname{ad}_{k} A(t)-\cosh (\lambda t) A(t) \operatorname{ad}_{k}\right) \circ P_{p, \gamma(t)} .
$$

Differentiating again in the same way, we get

$$
\nabla_{k} \nabla_{k} \mu(\gamma(t))=\omega_{p} \circ Z_{t, k}^{2}(A(t)) \circ P_{p, \gamma(t)},
$$

where $Z_{t, k}(A(t))=\operatorname{ad}_{k} A(t)-\cosh (\lambda t) A(t) \operatorname{ad}_{k}$. Thus,

$$
\Delta \mu(\gamma(t))=-\omega_{p} \circ\left(A^{\prime \prime}(t)+h(t) A^{\prime}(t)+\sum_{i=1}^{n} \frac{Z_{t, k_{i}}^{2}(A(t))}{\sinh \left(\lambda_{i} t\right)^{2} / \lambda_{i}^{2}}-A(t) \mathscr{R}\right) \circ P_{p, \gamma(t)} .
$$

Therefore,

$$
\Delta_{x}\left(\omega_{y} \circ q_{y, x}^{A}\right)=0
$$

holds for all $x=\gamma(t) \neq y=p=\gamma(0)$ and, by equivariance, for all $x, y, x \neq y$, provided that $A$ solves (22).

For the proof of (24), let $\alpha \in \Omega_{c}^{l}(X)$ be a $l$-form of compact support. For $\rho>0$, let $\phi \in C^{\infty}(X)$ be a bump function such that

$$
\phi(x)=\left\{\begin{array}{l}
1 \text { if } x \in B_{\rho / 2}(p) \\
0 \text { if } x \notin B_{\rho}(p)
\end{array}\right.
$$

and $|d \phi|<10 / \rho$. Then,

$$
\int_{X}\left\langle\Delta_{x} \alpha_{x} \mid \omega_{p} \circ q_{p, x}^{A}\right\rangle d x=\int_{X}\left\langle\Delta_{x}(\phi \alpha)_{x} \mid \omega_{p} \circ q_{p, x}^{A}\right\rangle d x+\int_{X}\left\langle\Delta_{x}((1-\phi) \alpha)_{x} \mid \omega_{p} \circ q_{p, x}^{A}\right\rangle d x .
$$

The right summand becomes

$$
\int_{X \backslash B_{\rho / 2}(p)}\left\langle\Delta_{x}((1-\phi) \alpha)_{x} \mid \omega_{p} \circ q_{p, x}^{A}\right\rangle d x=\int_{X \backslash B_{\rho / 2}(p)}\left\langle((1-\phi) \alpha)_{x} \mid \Delta_{x}\left(\omega_{p} \circ q_{p, x}^{A}\right)\right\rangle d x=0
$$

by what we proved above. For the left summand, we compare the Laplace operator of $X$ with the Euclidean Laplace operator $\Delta^{0}$ in normal coordinates near $p$ and then use that $\omega_{p} \circ q_{p, x}^{A}$ asymptotically coincides with $N$, which in turn is a fundamental solution to the Euclidean Laplace operator.

To this end, let $e_{i}=\frac{\partial}{\partial x_{i}}, i=1 \ldots d=n+1$, be the coordinate vector fields of normal coordinates near $p$. The Riemannian connection $\nabla$ of $X$ near $p$ is then of the form $\nabla_{v}=v+\Gamma_{v}$ with a local one form $\Gamma$ with values in the endomorphism bundle of TX. Since the coordinates are normal, we have $\Gamma_{v}(p)=0$ for all $v \in T_{p} X$. For the second covariant derivative, we compute

$$
\begin{gathered}
\nabla_{v, v}^{2}=\nabla_{v} \nabla_{w}-\nabla_{\nabla_{v} w}=\left(D_{v}+\Gamma_{v}\right)\left(D_{w}+\Gamma_{w}\right)-D_{\Gamma_{v} w}-\Gamma_{\Gamma_{v} w} \\
=D_{v, w}^{2}+\Gamma_{v} D_{w}+\Gamma_{w} D_{v}-D_{\Gamma_{v} w}+\left(D_{v} \Gamma_{w}\right)+\Gamma_{v} \Gamma_{w}-\Gamma_{\Gamma_{v} w} .
\end{gathered}
$$

Thus, for any $\alpha \in \Omega^{l}(X)$, we have

$$
\nabla^{2} \alpha=D^{2} \alpha+F D \alpha+H \alpha
$$

with tensor fields $F$ and $H$ and $F(0)=0$. By the Weitzenböck formula, we also have such a formula for the Laplacians, i.e.,

$$
\Delta \alpha=\Delta^{0} \alpha+F D \alpha+H \alpha
$$

with $F(p)=0$. In the sequel for $x \in B_{\rho}(p)$, we will write $r=r(x)=d(x, p)$. By our assumption, we have

$$
\omega_{p} \circ q_{p, x}^{A}=N \omega_{p}+R
$$

with some $R \in \Omega^{l}(X)$ of order $R \sim r^{2-n}$.

The left summand in (26) equals

$$
=\int_{B_{\rho}(p)}\left\langle\Delta_{x}(\phi \alpha)_{x} \mid \omega_{p} \circ q_{p, x}^{A}\right\rangle d x
$$


We will denote by $d x$ the volume form of the Riemannian metric from $X$ and by $d x^{0}$ the Euclidean volume form on $B_{\rho}(p)$ induced by the normal coordinates. If $g$ denotes the metric tensor expressed in the normal coordinates, then

$$
d x=\sqrt{\operatorname{det} g} d x^{0} \sim d x^{0}
$$

because $\operatorname{det} g(p)=1$. The integral (27) becomes

$$
\begin{aligned}
& =\int_{B_{\rho}(p)}\left\langle\Delta_{x}^{0}(\phi \alpha)_{x}+F(x) D(\phi \alpha)_{x}+H(x)(\phi \alpha)_{x} \mid N \omega_{p}+R\right\rangle d x \\
& =\int_{B_{\rho}(p)} \underbrace{\Delta_{x}^{0}(\phi \alpha)_{x}}_{\rho^{-2}}+\underbrace{F(x) D(\phi \alpha)_{x}}_{\rho \times \frac{1}{\rho}=\rho^{0}}+\underbrace{H(x)(\phi \alpha)_{x}}_{\rho^{0}}|\underbrace{N \omega_{p}}_{\rho^{1-n}}+\underbrace{R}_{\rho^{2-n}}| \underbrace{\sqrt{\operatorname{det} g}}_{1} d x^{0} .
\end{aligned}
$$

In the limit $\rho \rightarrow 0$, the terms involving $F, H$, and $R$ vanish, and thus for the integral (27), we finally get

$$
\begin{aligned}
& =\int_{X}\left\langle\Delta_{x} \alpha_{x} \mid \omega_{p} \circ q_{p, x}^{A}\right\rangle d x \\
& =\lim _{\rho \rightarrow 0} \int_{B_{\rho}(p)}\left\langle\Delta_{x}^{0}(\phi \alpha)_{x} \mid N \omega_{p}\right\rangle d x^{0} \\
& =\lim _{\rho \rightarrow 0}\left\langle\phi(p) \alpha_{p} \mid \omega_{p}\right\rangle=\left\langle\alpha_{p} \mid \omega_{p}\right\rangle,
\end{aligned}
$$

since $N$ is a fundamental solution for the Euclidean Laplacian.

\section{A. Existence of a solution}

A solution $A$ of Eq. (22) in Theorem 1 always exists. To see this, we rewrite the differential Eq. (22) in the form

$$
\left(\sigma A^{\prime}\right)^{\prime}=W(\sigma A),
$$

where WA denotes the right hand side of (22). Substituting

$$
z=\frac{\sigma A}{t} \text { and } y=\sigma A^{\prime}
$$

this becomes

$$
\begin{aligned}
& z^{\prime}=-\frac{z}{t}+\frac{\sigma A^{\prime}}{t}+\frac{\sigma^{\prime} A}{t}=\frac{1}{t}(y+(t h-1) z), \\
& y^{\prime}=W \sigma A=t W z .
\end{aligned}
$$

From (15) and (22), we can write $h$ and $W$ in the form

$$
h(t)=\frac{n}{t}+t h_{0}(t) \quad \text { and } \quad W(t)=\frac{W_{-2}}{t^{2}}+W_{0}(t)
$$

with even analytic functions $h_{0}$ and $W_{0}$. We thus have the regular singular first order differential equation,

$$
\begin{aligned}
\left(\begin{array}{l}
z \\
y
\end{array}\right)^{\prime} & =\frac{1}{t}\left(\begin{array}{cc}
\operatorname{th}(t)-1 & 1 \\
t^{2} W(t) & 0
\end{array}\right)\left(\begin{array}{l}
z \\
y
\end{array}\right) \\
& =\left(\frac{1}{t}\left(\begin{array}{cc}
n-1 & 1 \\
W_{-2} & 0
\end{array}\right)+t\left(\begin{array}{ll}
h_{0}(t) & 0 \\
W_{0}(t) & 0
\end{array}\right)\right)\left(\begin{array}{l}
z \\
y
\end{array}\right) .
\end{aligned}
$$

We now substitute $t=e^{-s}, Y(s)=(z, y)\left(e^{-s}\right)$, and $Y^{\prime}=-e^{-s}(z, y)^{\prime}\left(e^{-s}\right)$ to transform this into

$$
Y^{\prime}(s)=\left(-\left(\begin{array}{cc}
n-1 & 1 \\
W_{-2} & 0
\end{array}\right)-e^{-2 s}\left(\begin{array}{cc}
h_{0}\left(e^{-s}\right) & 0 \\
W_{0}\left(e^{-s}\right) & 0
\end{array}\right)\right) Y(s)
$$


Since $W_{-2}$ is Hermitian and nonnegative, we may assume that $W_{-2}$ is a diagonal matrix with nonnegative eigenvalues $w_{i}$, $i=1, \ldots, \operatorname{dim} \operatorname{Hom}_{H}\left(\Lambda^{l} \mathfrak{m}, \Lambda^{l} \mathfrak{m}\right)$. We can, therefore, diagonalize the matrix $\left(\begin{array}{cc}n-1 & 1 \\ W_{-2} & 0\end{array}\right)$ with $(2 \times 2)$-blocks of the form $\left(\begin{array}{cc}n-1 & 1 \\ w_{i} & 0\end{array}\right)$. Since the $w_{i}$ are nonnegative, these blocks have 2 different eigenvalues. It follows that they all can be diagonalized.

Therefore, Eq. (28) satisfies the conditions of the Levinson theorem for asymptotic constant (diagonalizable) coefficient systems.

Theorem 2 (Theorem 1.8.1 in Ref. 8). Let $C$ be a diagonalizable $m \times m$ matrix and $R(s)$ a matrix valued function, $s \in \mathbb{R}$. Assume that

$$
\int_{a}^{\infty}\|R(s)\| d s<\infty
$$

for all $a \in \mathbb{R}$. If $\left\{u_{k} \mid 1 \leq k \leq m\right\}$ are linearly independent eigenvectors of $C$ corresponding to the eigenvalues $\lambda_{k}, 1 \leq k \leq m$, then the differential equation

$$
Y^{\prime}(s)=(C+R(s)) Y(s)
$$

has solutions with the asymptotic form,

$$
Y_{k}(s)=\left(u_{k}+o(1)\right) e^{\lambda_{k} s} \quad \text { as } \quad s \rightarrow \infty
$$

In particular, for the eigenvalue 0 of $C=\left(\begin{array}{cc}n-1 & 1 \\ -W_{-2} & 0\end{array}\right)$, we get a solution $Y(s)=\left(\begin{array}{c}\frac{-1}{n-1} \mathrm{id} \\ \text { id }\end{array}\right)+o(1)$ for $(28)$, which corresponds to a solution $A$ for (22) with

$$
\sigma(t) A^{\prime}(t)=\mathrm{id}+o(1), \quad \frac{\sigma(t) A(t)}{t}=\frac{-\mathrm{id}}{n-1}+o(1) .
$$

Hence, because $\sigma(t)=t^{n}\left(\operatorname{vol} S^{n}+o(1)\right)$,

$$
A(t)=\frac{-t}{(n-1) \sigma(t)}(\mathrm{id}+o(1))=\frac{-t^{1-n}}{(n-1) \mathbf{v o l} S^{n}}(\mathrm{id}+o(1))
$$

as $t \rightarrow 0$.

The operators $d, d^{*}$, and $\Delta$ preserve this class of kernels. For later reference, we note their action on the corresponding radial function $A$.

Lemma 4. Let $A \in C^{\infty}\left(\mathbb{R}^{+}, \operatorname{Hom}_{H}\left(\Lambda^{k} \mathfrak{m}, \Lambda^{l} \mathfrak{m}\right)\right)$ and $Q^{A}$ be the integral operator with kernel $q^{A}$, i.e.,

$$
\left(Q^{A} \omega\right)_{x}=\int_{X} \omega_{y} \circ q_{y, x}^{A} d y
$$

for $\omega \in \Omega^{l}(X)$. Then, $d Q^{A}=Q^{d A}$ and $d^{*} Q^{A}=Q^{d^{*} A}$ with

$$
\begin{gathered}
d A \in C^{\infty}\left(\mathbb{R}^{+}, \operatorname{Hom}_{H}\left(\Lambda^{k+1} \mathfrak{m}, \Lambda^{l} \mathfrak{m}\right)\right), \\
d^{*} A \in C^{\infty}\left(\mathbb{R}^{+}, \operatorname{Hom}_{H}\left(\Lambda^{k-1} \mathfrak{m}, \Lambda^{l} \mathfrak{m}\right)\right)
\end{gathered}
$$

given by

$$
\begin{gathered}
d A(t)=T \wedge A^{\prime}(t)+\sum_{i} \frac{m_{i}(p) \wedge\left(\operatorname{ad}_{k_{i}} A(t)-\cosh \left(\lambda_{i} t\right) A(t) \operatorname{ad}_{k_{i}}\right)}{\sinh \left(t \lambda_{i}\right) / \lambda_{i}}, \\
-d^{*} A(t)=T \neg A^{\prime}(t)+\sum_{i} \frac{\left.m_{i}(p) \neg\left(\operatorname{ad}_{k_{i}} A(t)-\cosh \left(\lambda_{i} t\right) A(t) \operatorname{ad}_{k_{i}}\right)\right)}{\sinh \left(t \lambda_{i}\right) / \lambda_{i}} .
\end{gathered}
$$

Proof. This follows from (4) by the same calculation as in the Proof of Theorem 1.

\section{B. Hyperbolic space}

In space forms $X^{m}$, the orthogonal complement $\mathfrak{m}_{0}$ of the unit vector $T \in T_{p} X=\mathfrak{m}$ is irreducible for $H$. We have a decomposition

$$
\Lambda^{l} \mathfrak{m}=T \wedge \Lambda^{l-1} \mathfrak{m}_{0} \oplus \Lambda^{l} \mathfrak{m}_{0}
$$

as modules for $H$. For $A \in C^{\infty}\left(\mathbb{R}^{+}, \operatorname{Hom}_{H}\left(\Lambda^{l} \mathfrak{m}, \Lambda^{l} \mathfrak{m}\right)\right)$, we can, therefore, make the ansatz

$$
A(t)\left(T \wedge \mu_{0}+\mu_{1}\right)=\alpha(t) T \wedge \mu_{0}+\beta(t) \mu_{1}
$$


with

$$
\mu_{0} \in \Lambda^{l-1} \mathfrak{m}_{0}, \mu_{1} \in \Lambda^{l} \mathfrak{m}_{0} \quad \text { and } \quad \alpha, \beta \in C^{\infty}\left(\mathbb{R}^{+}, \mathbb{R}\right)
$$

Let $k=m-l=n+1-l$. A straightforward calculation shows that in hyperbolic $m$-space the curvature endomorphism $\mathscr{R}$ on $\Lambda^{l} \mathfrak{m}$ from the Weitzenböck formula is multiplication with $-k l$ and

$$
\operatorname{ad}_{k_{i}} m_{i}=-T, \quad \operatorname{ad}_{k_{i}} T=m_{i}, \quad \text { and } \quad \operatorname{ad}_{k_{i}} m_{j}=0 \quad \text { if } j \neq i .
$$

The differential equation (22) for $A(t)$ becomes

$$
\begin{aligned}
& \alpha^{\prime \prime}(t)+n \operatorname{coth}(t) \alpha^{\prime}(t)=-k l \alpha(t)+k \frac{1+\cosh (t)^{2}}{\sinh (t)^{2}} \alpha(t)-2 k \frac{\cosh (t)}{\sinh (t)^{2}} \beta(t), \\
& \beta^{\prime \prime}(t)+n \operatorname{coth}(t) \beta^{\prime}(t)=-k l \beta(t)+l \frac{1+\cosh (t)^{2}}{\sinh (t)^{2}} \beta(t)-2 l \frac{\cosh (t)}{\sinh (t)^{2}} \alpha(t) .
\end{aligned}
$$

The solution to (31) satisfying the initial condition (23) will be Laurent series $\alpha, \beta$ starting with $\frac{t^{1-n}}{(n-1) \mathbf{v o l} S^{n}}$, whose higher order terms can be computed from (31). The expressions of these solutions to (31) in terms of elementary functions are quite complicated. For example, in the case of hyperbolic 3 -space and the Laplacian on 1 -forms, $l=1, k=2$, we get solutions

$$
\begin{aligned}
& \alpha(t)=\frac{(2 t+1) \sinh (t)-\left(t^{2}+t\right) \cosh (t)}{4 \pi \sinh (t)^{3}}, \\
& \beta(t)=\frac{3 \sinh (t)^{2}-(\cosh (t)+2 t) \sinh (t)+t^{2}+t}{8 \pi \sinh (t)^{3}}-\frac{t}{4 \pi(\cosh (t)+1)},
\end{aligned}
$$

which have also been found in Ref. 5. In fact, $\alpha, \beta$ above are given by $\alpha(t)=-\phi_{2}(t)-\phi_{3}^{\prime \prime}(t)$ and $\beta(t)=-\phi_{2}(t)-\phi_{3}^{\prime}(t) / \sinh (t)$, where the functions $\phi_{2}, \phi_{3}$ are as in Ref. 5, Theorem 3, (3) p. 20.

\section{THE BIOT-SAVART FORMULA AND THE LINKING NUMBER}

By Hodge theory, on any oriented compact Riemannian manifold, a right inverse $Q$ of the Laplace operator on differential forms provides a right inverse to the Cartan differential $d$ and the codifferential $d^{*}$ simultaneously. Thus, let $Q$ be a right inverse of the Laplacian, i.e., $\Delta Q \omega=\omega$ for all $l$-forms $\omega$ in the image of $\Delta$. If, in particular, $\alpha \in \Omega^{l}(X)$ is exact, then $\alpha=d d^{*} Q \alpha$ and $d^{*} d^{*} Q \alpha=0$. Following Refs. 3, 5, and 6, we call an operator $B$ so that $d B \alpha=\alpha$ and $d^{*} B \alpha=0$ a Biot-Savart operator. This is motivated by the analogy of these equations with the Maxwell equations for the magnetic field $B$ caused by a stationary current $\alpha$.

The differential equation (22) is invariant under complex rescaling of the metric on $X$. If $A(t)$ solves (22) for the symmetric space whose Jacobi operator has eigenvalues $\lambda_{1}^{2}, \ldots, \lambda_{n}^{2}$, then for $\alpha \in \mathbb{C} \backslash\{0\}$,

$$
A_{\alpha}(t)=\alpha^{n-1} A(\alpha t)
$$

solves (22) and (23) with $\lambda_{i}$ replaced by $\alpha \lambda_{i}$ and the curvature tensor $R$ replaced by $\alpha^{2} R$. In particular, $A_{i}(t)=i^{n-1} A(i t)$ solves (22) for the compact dual $X_{c}$ of $X$. Since $A$ is holomorphic on $\mathbb{C} \backslash\{0\}, A_{\alpha}(t)$ is holomorphic in $\alpha$.

On the compact Riemannian manifold $X_{c}$, Hodge theory gives an $L^{2}$-orthogonal splitting of $\Omega^{l}\left(X_{c}\right)$ as in the diagram

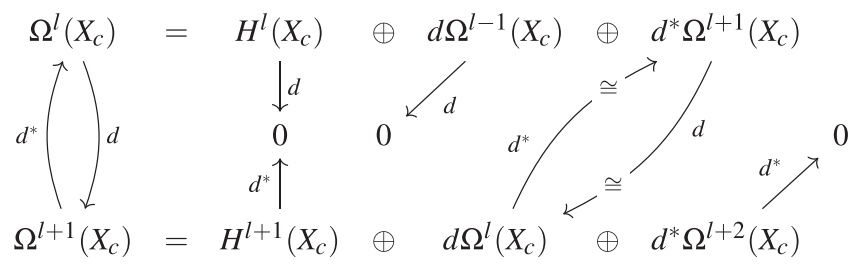


The Cartan differential $d$ and its adjoint restrict to isomorphisms as indicated. The Laplacian $\Delta_{c}=d d^{*}+d^{*} d$ of $X_{c}$ preserves the above splitting, vanishes on $H^{l}\left(X_{c}\right)=\operatorname{ker} \Delta_{c}=\operatorname{ker} d \cap \operatorname{ker} d^{*}$, and is isomorphic on both $d \Omega^{l-1}$ and $d^{*} \Omega^{l+1}$.

Let $\Delta_{c}^{-1}$ be the extension of the inverse of this isomorphism by 0 to all of $\Omega^{l}\left(X_{c}\right)$. By equivariance, the integral kernel of $\Delta_{c}^{-1}$ must be of the form (21) with a function $\tilde{A}_{c}$ satisfying (22) and (23) for the compact rank one symmetric space $X_{c}$. We can therefore choose a solution $A$ to (22) with $A_{i}=\tilde{A}_{c}$. In this section, $A$ will always be such a solution of (22) and (23).

Let $\omega \in d \Omega_{c}^{l}(X)$. Then, $\omega=d d^{*} \Delta_{c}^{-1} \omega=d Q^{d^{*} A_{i}} \omega=d Q^{d^{*} A_{i \alpha}} \omega$ for all $\alpha \in \mathbb{R}^{+}$. Since this is holomorphic in $\alpha$, the identity holds for all $\alpha \in$ $\mathbb{C} \backslash\{0\}$. Thus, $\omega=d Q^{d^{*} A} \omega$ for the above choice of $A$. We thus have the following theorem for the Biot-Savart operator on noncompact rank one symmetric spaces.

Theorem 3. Let $X$ be a noncompact rank one symmetric space and let $\omega \in \Omega_{c}^{l}(X)$ be a closed l-form of compact support. Then,

$$
B=d^{*} \Delta^{-1} \omega=\int_{X} \omega_{y} \circ q_{y, x}^{d^{*} A} d y
$$

satisfies

$$
d B=\omega \quad \text { and } \quad d^{*} B=0 .
$$

There is also an integral formula for the linking number similar to the classical Gauss formula for the linking number of loops in $\mathbb{R}^{3}$.

Theorem 4. Let $X^{d}$ be a noncompact rank one symmetric space. Let $K^{k}, L^{l} \subset X^{d}, k+l+1=d$ be disjoint closed submanifolds of dimensions $k$ and $l$, respectively. Let $A$ be the kernel function inverting the Laplace Beltrami operator on $k$-forms on $X$. Then, the linking number of $K$ and $L$ is given by

$$
L(K, L)=\int_{K} \int_{L} \mathbf{v o l}^{L}(y) \wedge q_{y, x}^{d^{*} A} \mathbf{v o l}^{K}(x) d y d x,
$$

where $\mathbf{v o l}^{L}(y)$ and $\mathbf{v o l}^{K}(x)$ denote the metric volume elements of $L$ at $y \in L$ and of $K$ at $x \in K$, respectively.

Proof. Let $K^{k}, L^{l} \subset X^{d}, k+l+1=d$ be disjoint closed submanifolds of dimensions $k$ and $l$, respectively, in a $d$-dimensional manifold $X$. Let $i: K \hookrightarrow X$ and $j: L \hookrightarrow X$ be the inclusions. We assume that $H_{c}^{d-k}(X) \cong H_{k}(X)=0=H_{c}^{d-l}(X) \cong H_{l}(X)$. Following the notation of Ref. 2, we let $\eta_{K} \in \Omega_{c}^{d-k}(X)$ be the Poincaré dual of $K$, i.e., a compactly supported form on $X$ such that

$$
d \eta_{K}=0 \quad \text { and } \quad \int_{K} i^{*} \alpha=\int_{X} \alpha \wedge \eta_{K}
$$

for all $\alpha \in \Omega^{k}(X)$ with $d \alpha=0$. Then, the linking number of $K$ and $L$ in $X$ is

$$
L(K, L):=\int_{X} \beta \wedge \eta_{K}=\int_{K} i^{*} d^{-1} \eta_{L},
$$

where $\beta$ is any form of degree $d-l=k+1$ with $d \beta=\eta_{L}$, for instance, $\beta=d^{*} \Delta^{-1} \eta_{L}=Q^{d^{*} A} \eta_{L}$,

$$
\beta_{x}=\int_{X} \eta_{L, y} \circ q_{y, x}^{d^{*} A} d y
$$

we obtain

$$
L(K, L)=\int_{K} i^{*} d^{*} \Delta^{-1} \eta_{L}=\int_{K} \int_{X} \eta_{L, y}\left(q_{y, x}^{d^{*} A} \mathbf{v o l}{ }^{K}(x)\right) d y d x
$$

where $\operatorname{vol}^{K}(x)$ denotes the metric volume element of $K$ at $x$. Let $N L$ be some tubular neighborhood of $L$ induced by normal coordinates. Thus, we choose $r>0$ so small that the Riemannian exponential map

$$
D_{r} v(L, X) \rightarrow N L, \quad v \in v_{y}(L, X) \mapsto \exp _{y} v
$$

is a diffeomorphism on the $r$-disk bundle of the normal bundle $v(L, X)$ of $L$ in $X$. For $1 \geq \rho>0$, let $N_{\rho} L$ denote the tubular neighborhood of $L$ obtained from $N L$ be rescaling with $\rho$, that is,

$$
N_{\rho} L=\{\rho v \mid v \in N L\} \subset N L .
$$


To represent the Poincare dual of the submanifold $L$, we may take the extension by 0 of the Thom form of the normal bundle of $L$. This is a closed $(k+1)$-form on $N L$ vanishing near the boundary of $N L$ and such that its integral over the fibers of $N L$ is always 1 . Let $\chi^{\rho}$ be the diffeomorphism

$$
\chi_{\rho}: N_{\rho} L \rightarrow N L, \quad v \mapsto \frac{1}{\rho} v
$$

and $\eta_{L}^{\rho} \in \Omega(X)$ be the extension by zero of

$$
\eta_{L}^{\rho}:=\chi_{\rho}^{*} \eta_{L}
$$

The forms $\eta_{L}^{\rho}$ are all closed. Since the diffeomorphisms $\chi_{\rho}$ preserve the fibers of the tubular neighborhoods, the integral over the fibers of $N L \rightarrow L$ of $\eta_{L}^{\rho}$ is always 1 . Thus, the forms $\eta_{L}^{\rho}$ are all Thom forms for the normal bundle, or, Poincaré duals of $L$. For the linking number, it follows that

$$
L(K, L)=\int_{K} \int_{N_{\rho} L} \eta_{L, y}^{\rho}\left(q_{y, x}^{d^{*} A} \mathbf{v o l}^{K}(x)\right) d y d x
$$

for all $1 \geq \rho>0$. We now take the limit $\rho \rightarrow 0$. Using the Riemannian metric, we split the tangent bundle of $N L$ into vertical and horizontal distributions. The Thom form $\eta_{L}$ can then be written as a sum

$$
\eta_{L}=\omega_{0}+\sum_{i=1}^{l} \alpha_{i} \wedge \omega_{i}
$$

with vertical forms $\omega_{i}$ of degree $k+1-i=d-l-i$ and horizontal forms $\alpha_{i}$ of degree $i . \omega_{0}$ is a volume form of the fibers. As $\rho \rightarrow 0$,

$$
\left(\chi^{\rho}\right)^{*}\left(\sum_{i=1}^{l} \alpha_{i} \wedge \omega_{i}\right) \rightarrow 0 \quad \text { in } \quad L^{1}
$$

because the differential of $\chi^{\rho}$ on $\omega_{i}$ scales with a factor of $1 / \rho^{k+1-I}$, but the support scales with $\rho^{k}$. Taking the limit $\rho \rightarrow 0$ in (33) gives

$$
\begin{aligned}
L(K, L)= & \lim _{\rho \rightarrow 0} \int_{K} \int_{N^{\rho} L}\left(\chi^{\rho}\right)^{*} \omega_{0, y}\left(q_{y, x}^{d^{*} A} \operatorname{vol}^{K}(x)\right) d y d x \\
& =\int_{K} \int_{L} \tilde{\omega}_{0, y}\left(q_{y, x}^{d^{*} A} \operatorname{vol}^{K}(x)\right) d y d x,
\end{aligned}
$$

where $\tilde{\omega}_{0} \in \Gamma\left(\left.\Lambda^{k+1} T X\right|_{L} ^{*}\right)$ is such that $\tilde{\omega}_{0, y}\left(v_{0}, v_{1}, \ldots v_{k}\right)=1$ if $\left(v_{0}, v_{1}, \ldots v_{k}\right)$ is an oriented orthonormal basis for the orthogonal complement of $T_{y} L$ in $T_{y} X$ and 0 if one of the $v_{i}$ is tangent to $L$. Thus, the above integral becomes

$$
=\int_{K} \int_{L} \mathbf{v o l}^{L}(y) \wedge q_{y, x}^{d^{*} A} \operatorname{vol}^{K}(x) d y d x
$$

\section{ACKNOWLEDGEMENTS}

We are grateful to Marcos Salvai for initiating this work, for many profitable discussions during his visit to the University of Cyprus, and for bringing to our attention Refs. 3 and $5-7$. We also thank the referee for valuable comments.

\section{REFERENCES}

${ }^{1}$ A. L. Besse, Einstein Manifolds, Ergebnisse der Mathematik und ihrer Grenzgebiete (Springer-Verlag, Berlin, 1987), Vol. 10, pp. xii+510.

${ }^{2}$ R. Bott and L. W. Tu, Differential Forms in Algebraic Topology, Graduate Texts in Mathematics (Springer-Verlag, New York, Berlin, 1982), Vol. 82, pp. xiv+331.

${ }^{3}$ J. Cantarella, D. DeTurck, and H. Gluck, “The Biot-Savart operator for application to knot theory, fluid dynamics and plasma physics,” J. Math. Phys. 42, 876-905 (2001).

${ }^{4}$ E. Damek and F. Ricci, “A class of nonsymmetric harmonic Riemannian spaces,” Bull. Am. Math. Soc.27, 139-142 (1992).

${ }^{5}$ D. DeTurck and H. Gluck, "Electrodynamics and the Gauss linking integral on the 3-sphere and in hyperbolic 3-space," J. Math. Phys. 49, 023504 (2008).

${ }^{6}$ D. DeTurck and H. Gluck, "Linking, twisting, writhing, and helicity on the 3-sphere and in hyperbolic 3-space,” J. Differ. Geom. 94(1), 87-128 (2013).

${ }^{7}$ D. DeTurck and H. Gluck, "Linking integrals in the $n$-sphere," Mat. Comtemp. 34, 239-249 (2008).

${ }^{8}$ M. S. P. Eastham, "The asymptotic solution of linear differential systems," in Applications of the Levinson Theorem, London Mathematical Society Monographs New Series (Oxford Science Publications; The Clarendon Press; Oxford University Press, New York, 1989), Vol. 4, pp. x+241.

${ }^{9}$ J.-H. Eschenburg, Lecture Notes on Symmetric Spaces, https://www.researchgate.net/publication/241529230_Lecture_Notes_on_Symmetric_Spaces. 
${ }^{10}$ S. Helgason, Differential Geometry, Lie Groups and Symmetric Spaces (Academic Press, 1978).

${ }^{11}$ G. Kuperberg, "From the Mahler conjecture to Gauss linking integrals," Geom. Funct. Anal. 18, 870-892 (2008).

${ }^{12}$ H. B. Lawson and M. Michelsohn, Spin Geometry, Princeton Mathematical Series (Princeton University Press, Princeton, NJ, 1989), Vol. 38, pp. xii+427

${ }^{13}$ A. Lichnerowicz, "Sur les espaces riemanniem completement harmoniques," Bull. Soc. Math. Fr. 72, 146-168 (1944).

${ }^{14}$ O. Loos, Symmetric Spaces I, II (Benjamin, 1969).

${ }^{15}$ Z. I. Szabó, "The Lichnerowicz conjecture on harmonic manifolds," J. Differ. Geom. 31(1), 1-28 (1990), Inventiones mathematicae. 